K. SZA J OWSKI (Wrocław)

\title{
A TWO-DISORDER DETECTION PROBLEM
}

Abstract. Suppose that the process $X=\left\{X_{n}, n \in \mathbb{N}\right\}$ is observed sequentially. There are two random moments of time $\theta_{1}$ and $\theta_{2}$, independent of $X$, and $X$ is a Markov process given $\theta_{1}$ and $\theta_{2}$. The transition probabilities of $X$ change for the first time at time $\theta_{1}$ and for the second time at time $\theta_{2}$. Our objective is to find a strategy which immediately detects the distribution changes with maximal probability based on observation of $X$. The corresponding problem of double optimal stopping is constructed. The optimal strategy is found and the corresponding maximal probability is calculated.

1. Introduction. Suppose that a process $X=\left\{X_{n}, n \in \mathbb{N}\right\}(\mathbb{N}=$ $\{0,1,2, \ldots\})$ is observed sequentially. The process is obtained from three Markov processes by switches between them at two random moments of time, $\theta_{1}$ and $\theta_{2}$. Our objective is to detect immediately these moments based on observation of $X$.

This type of problem arises in quality control. An automaton which produces some details changes its parameters. This causes the details to change their quality. Production can be divided into three grades. Assuming that at the beginning of the production process the quality is highest, from some time $\theta_{1}$ on the products should be classified to a lower grade, and beginning with $\theta_{2}$ to the lowest grade. We want to detect the moments of these changes.

Shiryaev (1978) considered the disorder problem for independent random variables with one disorder where the mean distance between disorder time and the moment of its detection was minimized. The probability maximizing approach to the problem was used by Bojdecki (1979), and the stopping time which is in a given neighbourhood of the moment of disorder with

1991 Mathematics Subject Classification: Primary 60G40; Secondary 62L15.

Key words and phrases: disorder problem; sequential detection; multiple optimal stopping. 
maximal probability was found. The problem with two disorders was considered by Yoshida (1983) and Szajowski (1992). Yoshida (1983) solved the problem of optimal stopping for the observation of a Markov process $X$ so as to maximize the probability that the distance between $\theta_{i}, i=1,2$, and the moment of disorder will not exceed a given number (for each disorder independently). He constructed a strategy which stops the process between the first and the second disorder with maximal probability. References to other papers treating variations of the disorder problem can be found in Szajowski (1992).

In the present paper the probability maximizing approach to optimal stopping developed by Bojdecki (1979) is extended to solve a double stopping problem (see Haggstrom (1967), Nikolaev (1979)) arising in the quickest detection of double disorders. In Section 2 the problem is formulated in a rigorous manner. Section 3 contains the reduction of the problem to an optimal stopping problem for a doubly indexed stochastic sequence. The main result is given in Section 4.

2. The double disorder detection problem. Let $X=\left\{X_{n}, n \in \mathbb{N}\right\}$, defined on $(\Omega, \mathcal{F}, P)$, be a potentially observable sequence of r.v.'s with values in $(\mathbb{E}, \mathcal{B})$, where $\mathbb{E}$ is a subset of the real line. Assume that the epochs of distributional changes are $\mathbb{N}$-valued $\mathcal{F}$-measurable r.v. $\theta_{1}$ and $\theta_{2}$, independent of $X$ and having the distribution

$$
P\left(\theta_{1}=j\right)=p_{1}^{j-1} q_{1}, \quad P\left(\theta_{2}=k \mid \theta_{1}=j\right)=p_{2}^{k-j-1} q_{2},
$$

where $j=1,2, \ldots, k=j+1, j+2, \ldots$ and $p_{i}+q_{i}=1, i=1,2$.

Suppose that on $(\Omega, \mathcal{F}, P)$ Markov processes $X^{i}=\left\{\left(X_{n}^{i}, \mathcal{F}_{n}, P_{x}^{i}\right)\right\}, i=$ $1,2,3$, are defined and we have

$$
X_{n}= \begin{cases}X_{n}^{1} & \text { if } n<\theta_{1} \\ X_{n}^{2} & \text { if } \theta_{1} \leq n<\theta_{2} \\ X_{n}^{3} & \text { if } n \geq \theta_{2}\end{cases}
$$

The measures $P_{x}^{i}, i=1,2,3$, are absolutely continuous with respect to some fixed measure $P_{x}$ and satisfy the following relations: $P_{x}^{i}(d y)=f_{x}^{i}(y) P(x, d y)$, where $f_{x}^{i}(\cdot) \neq f_{x}^{j}(\cdot), i \neq j$ and $f_{x}^{i+1}(y) / f_{x}^{i}(y)<\infty, i=1,2$, for every $x, y \in \mathbb{E}$. The distribution of $\theta_{i}, i=1,2$, is given by (1) and the measures $P_{x}^{i}, i=1,2,3, x \in \mathbb{E}$, are known. We observe the process $\left(X_{n}, \mathcal{F}_{n}, P_{x}\right)$, $n=0,1,2, \ldots, x \in \mathbb{E}$, which is a Markov process given $\theta_{1}$ and $\theta_{2}$, defined by (2) with $\mathcal{F}_{n}=\sigma\left(X_{0}, X_{1}, \ldots, X_{n}\right)$. On the basis of the distribution of $\theta_{1}$, $\theta_{2}$ and measures $P_{x}^{i}, i=1,2,3, x \in \mathbb{E}$, we calculate the finite-dimensional distributions of the observed process.

Let $\mathcal{S}$ denote the set of all stopping times with respect to the filtration $\left(\mathcal{F}_{n}\right), n=0,1, \ldots$, and $\mathcal{T}=\{(\tau, \sigma): \tau<\sigma, \tau, \sigma \in \mathcal{S}\}$. Let us determine a 
pair of stopping times $\left(\tau^{*}, \sigma^{*}\right) \in \mathcal{T}$ such that for every $x \in \mathbb{E}$,

$$
\begin{aligned}
P_{x}\left(\tau^{*}<\sigma^{*}\right. & \left.<\infty,\left|\theta_{1}-\tau^{*}\right| \leq d_{1},\left|\theta_{2}-\sigma^{*}\right| \leq d_{2}\right) \\
& =\sup _{(\tau, \sigma) \in \mathcal{T}} P_{x}\left(\tau<\sigma<\infty,\left|\theta_{1}-\tau\right| \leq d_{1},\left|\theta_{2}-\sigma\right| \leq d_{2}\right) .
\end{aligned}
$$

This problem will be denoted by $D_{d_{1} d_{2}}$.

3. Reduction of the double "disorder problem" to double optimal stopping of a Markov process. A compound stopping variable is a pair $(\tau, \sigma)$ of stopping times such that $\tau<\sigma$ a.e. Define $\mathcal{T}_{m}=\{(\tau, \sigma) \in \mathcal{T}$ : $\tau \geq m\}, \mathcal{T}_{m n}=\{(\tau, \sigma) \in \mathcal{T}: \tau=m, \sigma \geq n\}$ and $\mathcal{S}_{m}=\{\tau \in \mathcal{S}: \tau \geq m\}$. Set $\mathcal{F}_{m n}=\mathcal{F}_{n}, m, n \in \mathbb{N}, m \leq n$. We define a two-parameter stochastic sequence $\xi(x)=\left\{\xi_{m n}(x), m, n \in \mathbb{N}, m<n, x \in \mathbb{E}\right\}$ by

$$
\xi_{m n}(x)=P_{x}\left(\left|\theta_{1}-\tau\right| \leq d_{1},\left|\theta_{2}-\sigma\right| \leq d_{2} \mid \mathcal{F}_{m n}\right) .
$$

For every $m, n \in \mathbb{N}$ with $m<n$, we can consider the optimal stopping problem of $\xi(x)$ on $\mathcal{T}_{m n}$. A compound stopping variable $\left(\tau^{*}, \sigma^{*}\right)$ is said to be optimal in $\mathcal{T}_{m}\left(\right.$ or $\left.\mathcal{T}_{m n}\right)$ if $E_{x} \xi_{\tau^{*} \sigma^{*}}=\sup _{(\tau, \sigma) \in \mathcal{T}_{m}} E_{x} \xi_{\tau \sigma}$ (or $E_{x} \xi_{\tau^{*} \sigma^{*}}=$ $\left.\sup _{(\tau, \sigma) \in \mathcal{T}_{m n}} E_{x} \xi_{\tau \sigma}\right)$. Define

$$
\begin{gathered}
\eta_{m n}(x)=\operatorname{ess~sup}_{(\tau, \sigma) \in \mathcal{T}_{m n}} E\left(\xi_{\tau \sigma} \mid \mathcal{F}_{m n}\right), \\
\eta_{m}=E_{x}\left(\eta_{m, m+1} \mid \mathcal{F}_{m}\right) .
\end{gathered}
$$

If we put $\xi_{m \infty}=0$, then

$$
\eta_{m n}=\operatorname{ess~sup}_{(\tau, \sigma) \in \mathcal{T}_{m n}} P_{x}\left(\left|\theta_{1}-m\right| \leq d_{1},\left|\theta_{2}-n\right| \leq d_{2} \mid \mathcal{F}_{m n}\right) .
$$

From the theory of optimal stopping for double indexed processes (cf. Haggstrom (1967), Nikolaev (1981)) the sequence $\eta_{m n}$ satisfies

$$
\eta_{m n}=\max \left\{\xi_{m n}, E\left(\eta_{m, n+1} \mid \mathcal{F}_{m n}\right)\right\} .
$$

Moreover, if $\sigma_{m}^{*}=\inf \left\{n \geq m: \eta_{m n}=\xi_{m n}\right\}$, then $\left(m, \sigma_{m}^{*}\right)$ is optimal in $\mathcal{T}_{m n}$ and $\eta_{m n}=E_{x}\left(\xi_{m \sigma_{m}^{*}} \mid \mathcal{F}_{m n}\right)$ a.e.

Lemma 1. The stopping time $\sigma_{m}^{*}$ is optimal for every stopping problem (4).

Proof. It suffices to prove $\lim _{n \rightarrow \infty} \xi_{m n}=0$ (Lemma 4.10 of Chow, Robbins \& Siegmund (1971), cf. also Bojdecki (1979), Bojdecki (1982)). For $m, n, k \in \mathbb{N}$ with $n \geq k>m$ and every $x \in \mathbb{E}$ we have

$$
\begin{aligned}
E_{x}\left(\mathbb{I}_{\left\{\left|\theta_{1}-m\right| \leq d_{1},\left|\theta_{2}-n\right| \leq d_{2}\right\}} \mid \mathcal{F}_{m n}\right) & =\xi_{m n}(x) \\
& \leq E_{x}\left(\sup _{j \geq k} \mathbb{I}_{\left\{\left|\theta_{1}-m\right| \leq d_{1},\left|\theta_{2}-j\right| \leq d_{2}\right\}} \mid \mathcal{F}_{n}\right),
\end{aligned}
$$


where $\mathbb{I}_{A}$ is the characteristic function of the set $A$. By Levy's theorem,

$$
\limsup _{n \rightarrow \infty} \xi_{m n}(x) \leq E_{x}\left(\sup _{j \geq k} \mathbb{I}_{\left\{\left|\theta_{1}-m\right| \leq d_{1},\left|\theta_{2}-j\right| \leq d_{2}\right\}} \mid \mathcal{F}_{n \infty}\right),
$$

where $\mathcal{F}_{\infty}=\mathcal{F}_{n \infty}=\sigma\left(\bigcup_{n=1}^{\infty} \mathcal{F}_{n}\right)$.

We have $\lim _{k \rightarrow \infty} \sup _{j \geq k} \mathbb{I}_{\left\{\left|\theta_{1}-m\right| \leq d_{1},\left|\theta_{2}-j\right| \leq d_{2}\right\}}=0$ a.e. and by the dominated convergence theorem,

$$
\lim _{k \rightarrow \infty} E_{x}\left(\sup _{j \geq k} \mathbb{I}_{\left\{\left|\theta_{1}-m\right| \leq d_{1},\left|\theta_{2}-j\right| \leq d_{2}\right\}} \mid \mathcal{F}_{\infty}\right)=0 .
$$

As the next step the optimal stopping problem for $\eta_{m}$ should be solved. Define

$$
V_{m}=\underset{\tau \in \mathcal{S}_{m}}{\operatorname{ess} \sup } E_{x}\left(\eta_{\tau} \mid \mathcal{F}_{m}\right) .
$$

Then $V_{m}=\max \left\{\eta_{m}, E_{x}\left(V_{m+1} \mid \mathcal{F}_{m}\right)\right\}$ a.e. and we define $\tau_{n}^{*}=\inf \{k \geq n$ : $\left.V_{k}=\eta_{k}\right\}$.

LEMMA 2. The strategy $\tau_{0}^{*}$ is the optimal strategy of the first stop.

Proof. To show that $\tau_{0}^{*}$ is the optimal first stop strategy we prove that $P_{x}\left(\tau_{0}^{*}<\infty\right)=1$. We argue in the usual manner, i.e. we show $\lim _{m \rightarrow \infty} \eta_{m}(x)$

$=0$.

We have

$$
\begin{aligned}
\eta_{m} & =E_{x}\left(\xi_{m \sigma_{m}^{*}} \mid \mathcal{F}_{m}\right)=E_{x}\left(E_{x}\left(\mathbb{I}_{\left\{\left|\theta_{1}-m\right| \leq d_{1},\left|\theta_{2}-\sigma_{m}^{*}\right| \leq d_{2}\right\}} \mid \mathcal{F}_{m \sigma_{m}^{*}}\right) \mid \mathcal{F}_{m}\right) \\
& =E_{x}\left(\mathbb{I}_{\left\{\left|\theta_{1}-m\right| \leq d_{1},\left|\theta_{2}-\sigma_{m}^{*}\right| \leq d_{2}\right\}} \mid \mathcal{F}_{m}\right) \\
& \leq E_{x}\left(\sup _{j \geq k} \mathbb{I}_{\left\{\left|\theta_{1}-j\right| \leq d_{1},\left|\theta_{2}-\sigma_{j}^{*}\right| \leq d_{2}\right\}} \mid \mathcal{F}_{m}\right) .
\end{aligned}
$$

Similarly to the proof of Lemma 1 we have

$$
\limsup _{m \rightarrow \infty} \eta_{m}(x) \leq E_{x}\left(\sup _{j \geq k} \mathbb{I}_{\left\{\left|\theta_{1}-j\right| \leq d_{1},\left|\theta_{2}-\sigma_{j}^{*}\right| \leq d_{2}\right\}} \mid \mathcal{F}_{\infty}\right) .
$$

Since

$$
\lim _{k \rightarrow \infty} \sup _{j \geq k} \mathbb{I}_{\left\{\left|\theta_{1}-k\right| \leq d_{1},\left|\theta_{2}-\sigma_{j}^{*}\right| \leq d_{2}\right\}} \leq \limsup _{k \rightarrow \infty} \mathbb{I}_{\left\{\left|\theta_{1}-k\right| \leq d_{1}\right\}}=0,
$$

it follows that

$$
\lim _{m \rightarrow \infty} \eta_{m}(x) \leq \lim _{k \rightarrow \infty} E_{x}\left(\sup _{j \geq k} \mathbb{I}_{\left\{\left|\theta_{1}-j\right| \leq d_{1},\left|\theta_{2}-\sigma_{j}^{*}\right| \leq d_{2}\right\}} \mid \mathcal{F}_{\infty}\right)=0 .
$$

Lemmas 1 and 2 describe the method of solving the "disorder problem" formulated in Section 2.

4. Immediate detection of the first and second disorder. For the sake of simplicity we restrict ourselves to the case $d_{1}=d_{2}=0$. It will be easily seen how to generalize the solution to $D_{d_{1} d_{2}}$ for $d_{1}>0$ or $d_{2}>0$. 
First we construct multi-dimensional Markov chains such that $\xi_{m n}$ and $\eta_{m}$ are the functions of their states. Set (cf. Yoshida (1983), Szajowski (1992))

$$
\begin{aligned}
& \Pi_{n}^{1}(x)=P_{x}\left(\theta_{1}>n \mid \mathcal{F}_{n}\right), \quad \Pi_{n}^{2}(x)=P_{x}\left(\theta_{2}>n \mid \mathcal{F}_{n}\right), \\
& \Pi_{m n}(x)=P_{x}\left(\theta_{1}=m, \theta_{2}>n \mid \mathcal{F}_{m n}\right) \quad \text { for } m, n=1,2, \ldots, m<n \text {, } \\
& H(t, u, \alpha, \beta)=\alpha p_{1} f_{t}^{1}(u)+\left[p_{2}(\beta-\alpha)+q_{1} \alpha\right] f_{t}^{2}(u) \\
& +\left[1-\beta+q_{2}(\beta-\alpha)\right] f_{t}^{3}(u), \\
& \Pi^{1}(t, u, \alpha, \beta)=p_{1} \alpha f_{t}^{1}(u)(H(t, u, \alpha, \beta))^{-1}, \\
& \Pi^{2}(t, u, \alpha, \beta)=\left\{p_{1} \alpha f_{t}^{1}(u)+\left[\alpha q_{1}+(\beta-\alpha) p_{2}\right] f_{t}^{2}(u)\right\}(H(t, u, \alpha, \beta))^{-1}, \\
& \Pi(t, u, \alpha, \beta, \gamma)=p_{2} \gamma f_{t}^{2}(u)(H(t, u, \alpha, \beta))^{-1} .
\end{aligned}
$$

The following auxiliary results will be needed in the proof of the main theorem.

Lemma 3. For each $x \in \mathbb{E}$ and $m, n=1,2, \ldots$ with $m<n$, and each Borel function $u: \mathbb{R} \rightarrow \mathbb{R}$,

$$
\begin{aligned}
\Pi_{n+1}^{1}(x) & =\Pi^{1}\left(X_{n}, X_{n+1}, \Pi_{n}^{1}(x), \Pi_{n}^{2}(x)\right), \\
\Pi_{n+1}^{2}(x) & =\Pi^{2}\left(X_{n}, X_{n+1}, \Pi_{n}^{1}(x), \Pi_{n}^{2}(x)\right), \\
\Pi_{m, n+1}(x) & =\Pi\left(X_{n}, X_{n+1}, \Pi_{n}^{1}(x), \Pi_{n}^{2}(x), \Pi_{m n}(x)\right),
\end{aligned}
$$

with the boundary condition $\Pi_{0}^{1}(x)=\Pi_{0}^{2}(x)=0$,

$$
\Pi_{m m}(x)=\frac{q_{1} f_{X_{m-1}}^{2}\left(X_{m}\right)}{p_{1} f_{X_{m-1}}^{1}\left(X_{m}\right)} \Pi_{n}^{1}(x)
$$

and

$$
E_{x}\left(u\left(X_{n+1}\right) \mid \mathcal{F}_{n}\right)=\int_{\mathbb{E}} u(y) H\left(X_{n}, y, \Pi_{n}^{1}(x), \Pi_{n}^{2}(x)\right) P_{X_{n}}(d y) .
$$

Proof. (7), (8) and (10) are proved in Yoshida (1983) and Szajowski (1992). The formula (9) follows from the Bayes formula:

$$
\begin{aligned}
P_{x}\left(\theta_{1}=j,\right. & \left.\theta_{2}=k \mid \mathcal{F}_{n}\right) \\
= & \left\{\begin{array}{cl}
P_{x}\left(\theta_{1}=j, \theta_{2}=k\right) p_{1}^{n} \prod_{s=1}^{n} f_{x_{s-1}}^{1}\left(x_{s}\right) & \\
\quad \times\left(S_{n}\left(x_{0}, x_{1}, \ldots, x_{n}\right)\right)^{-1} & \text { if } j>n, \\
P_{x}\left(\theta_{1}=j, \theta_{2}=k\right) \prod_{s=1}^{j-1} f_{x_{s-1}}^{1}\left(x_{s}\right) \prod_{t=j}^{n} f_{x_{t-1}}^{2}\left(x_{t}\right) & \\
\quad \times\left(S_{n}\left(x_{0}, x_{1}, \ldots, x_{n}\right)\right)^{-1} & \text { if } j \leq n<k, \\
P_{x}\left(\theta_{1}=j, \theta_{2}=k\right) \prod_{s=1}^{n} f_{x_{s-1}}^{1}\left(x_{s}\right) \prod_{t=j}^{k-1} f_{x_{t-1}}^{2}\left(x_{t}\right) & \\
\quad \times \prod_{u=k}^{n} f_{x_{u-1}}^{3}\left(x_{u}\right)\left(S_{n}\left(x_{0}, x_{1}, \ldots, x_{n}\right)\right)^{-1} & \text { if } k \leq n,
\end{array}\right.
\end{aligned}
$$


where

$$
\begin{aligned}
S_{n}\left(x_{0}, x_{1}, \ldots, x_{n}\right) & \\
= & \sum_{j=1}^{n-1} \sum_{k=j+1}^{n}\left\{p_{1}^{j-1} q_{1} p_{2}^{k-j-1} q_{2} \prod_{s=1}^{j-1} f_{x_{s-1}}^{1}\left(x_{s}\right) \prod_{t=j}^{k-1} f_{x_{t-1}}^{2}\left(x_{t}\right) \prod_{u=k}^{n} f_{x_{u-1}}^{3}\left(x_{u}\right)\right\} \\
& +\sum_{j=1}^{n}\left\{p_{1}^{j-1} q_{1} p_{2}^{n-j} \prod_{s=1}^{j-1} f_{x_{s-1}}^{1}\left(x_{s}\right) \prod_{t=j}^{n} f_{x_{t-1}}^{2}\left(x_{t}\right)\right\}+p_{1}^{n} \prod_{s=1}^{n} f_{x_{s-1}}^{1}\left(x_{s}\right) .
\end{aligned}
$$

We have

$$
\begin{aligned}
\Pi_{m, n+1}(x)= & P_{x}\left(\theta_{1}=m, \theta_{2}>n+1 \mid \mathcal{F}_{n+1}\right) \\
= & p_{2} f_{X_{n}}^{2}\left(X_{n+1}\right) \Pi_{m n}(x) S_{n}\left(x_{0}, x_{1}, \ldots, x_{n+1}\right) \\
& \times\left(S_{n+1}\left(x_{0}, x_{1}, \ldots, x_{n}\right)\right)^{-1}
\end{aligned}
$$

and

$$
S_{n+1}\left(x_{0}, x_{1}, \ldots, x_{n+1}\right)=H\left(X_{n}, X_{n+1}, \Pi_{n}^{1}(x), \Pi_{n}^{2}(x)\right) S_{n}\left(x_{0}, x_{1}, \ldots, x_{n}\right) .
$$

Hence

$$
\Pi_{m, n+1}(x)=\frac{p_{2} f_{X_{n}}^{2}\left(X_{n+1}\right) \Pi_{m n}(x)}{H\left(X_{n}, X_{n+1}, \Pi_{n}^{1}(x), \Pi_{n}^{2}(x)\right)} .
$$

By the above we have

$$
\begin{aligned}
\xi_{m n}(x)= & P_{x}\left(\theta_{1}=j, \theta_{2}=k \mid \mathcal{F}_{m n}\right) \\
= & \frac{p_{1}^{j-1} q_{1} p_{2}^{k-j-1} q_{2} \prod_{s=1}^{j-1} f_{x_{s-1}}^{1}\left(x_{s}\right) \prod_{t=j}^{n-1} f_{x_{t-1}}^{2}\left(x_{t}\right) f_{X_{n-1}}^{3}\left(X_{n}\right)}{S_{n}\left(x_{0}, x_{1}, \ldots, x_{n}\right)} \\
= & \frac{q_{2}}{p_{2}} \Pi_{m n}(x) \frac{f_{X_{n-1}}^{3}\left(X_{n}\right)}{f_{X_{n-1}}^{2}\left(X_{n}\right)} .
\end{aligned}
$$

We can observe that $\left(X_{n}, X_{n+1}, \Pi_{n}^{1}(x), \Pi_{n}^{2}(x), \Pi_{m n}(x)\right)$ for $n=m+1$, $m+2, \ldots$ is a function of $\left(X_{n-1}, X_{n}, \Pi_{n-1}^{1}(x), \Pi_{n-1}^{2}(x), \Pi_{m, n-1}(x)\right)$ and $X_{n+1}$. Moreover, the conditional distribution of $X_{n+1}$ given $\mathcal{F}_{n}$ (cf. (10)) depends on $X_{n}, \Pi_{n}^{1}(x)$ and $\Pi_{n}^{2}(x)$ only. These facts imply that $\left\{\left(X_{n}, X_{n+1}, \Pi_{n}^{1}(x), \Pi_{n}^{2}(x), \Pi_{m n}(x)\right)\right\}_{n=m+1}^{\infty}$ form a homogeneous Markov process (see Chapter 2.15 of Shiryaev (1978)). This allows us to reduce the problem (4) for each $m$ to the optimal stopping problem for the Markov process $Z_{m}(x)=\left\{\left(X_{n-1}, X_{n}, \Pi_{n}^{1}(x), \Pi_{n}^{2}(x), \Pi_{m n}(x)\right), m, n \in \mathbb{N}, m<n, x \in\right.$ $\mathbb{E}\}$ with the reward function

$$
h(t, u, \alpha, \beta, \gamma)=\frac{q_{2}}{p_{2}} \gamma \frac{f_{t}^{3}(u)}{f_{t}^{2}(u)} .
$$


Lemma 4. The solution of the optimal stopping problem (4) for $m=$ $1,2, \ldots$ has the form

$$
\sigma_{m}^{*}=\inf \left\{n>m: \frac{f_{X_{n-1}}^{3}\left(X_{n}\right)}{f_{X_{n-1}}^{2}\left(X_{n}\right)} \geq R^{*}\left(X_{n}\right)\right\}
$$

where $R^{*}(t)=p_{2} \int_{\mathbb{E}} r^{*}(t, s) f_{t}^{2}(s) P_{t}(d s)$. Here $r^{*}=\lim _{n \rightarrow \infty} r_{n}$, where $r_{0}(t, u)=f_{t}^{3}(u) / f_{t}^{2}(u)$ and

$$
r_{n+1}(t, u)=\max \left\{\frac{f_{t}^{3}(u)}{f_{t}^{2}(u)}, p_{2} \int_{\mathbb{E}} r_{n}(u, s) f_{u}^{2}(s) P_{u}(d s)\right\} .
$$

The function $r^{*}(t, u)$ satisfies the equation

$$
r^{*}(t, u)=\max \left\{\frac{f_{t}^{3}(u)}{f_{t}^{2}(u)}, p_{2} \int_{\mathbb{E}} r^{*}(u, s) f_{u}^{2}(s) P_{u}(d s)\right\} .
$$

The value of the problem is

$$
\eta_{m}=E_{x}\left(\eta_{m, m+1} \mid \mathcal{F}_{m}\right)=\frac{q_{2}}{p_{2}} \frac{q_{1}}{p_{1}} \Pi_{n}^{1}(x) R^{*}\left(X_{m}\right) .
$$

Proof. For any Borel function $u: \mathbb{E} \times \mathbb{E} \times[0,1]^{3} \rightarrow[0,1]$ define two operators

$$
\begin{array}{r}
T_{x} u(t, s, \alpha, \beta, \gamma)=E_{x}\left(u\left(X_{n}, X_{n+1}, \Pi_{n+1}^{1}(x), \Pi_{n+1}^{2}(x), \Pi_{m, n+1}(x)\right) \mid\right. \\
\left.X_{n-1}=t, X_{n}=s, \Pi_{n}^{1}(x)=\alpha, \Pi_{n}^{2}(x)=\beta, \Pi_{m n}(x)=\gamma\right)
\end{array}
$$

and

$$
Q_{x} u(t, s, \alpha, \beta, \gamma)=\max \left\{u(t, s, \alpha, \beta, \gamma), T_{x} u(t, s, \alpha, \beta, \gamma)\right\}
$$

By the well-known theorem from the theory of optimal stopping (see Shiryaev (1978), Ch. 2, and Nikolaev (1981)) we conclude that the solution of (4) is the Markov time

$$
\begin{aligned}
\sigma_{m}^{*}=\inf \left\{n>m: h\left(X_{n-1},\right.\right. & \left.X_{n}, \Pi_{n}^{1}(x), \Pi_{n}^{2}(x), \Pi_{m n}(x)\right) \\
& \left.=h^{*}\left(X_{n-1}, X_{n}, \Pi_{n}^{1}(x), \Pi_{n}^{2}(x), \Pi_{m n}(x)\right)\right\},
\end{aligned}
$$

where $h^{*}=\lim _{k \rightarrow \infty} Q_{x}^{k} h(t, u, \alpha, \beta, \gamma)$. Then

$$
\begin{aligned}
& T_{x} h(t, u, \alpha, \beta, \gamma)=E_{x}\left(\frac{q_{2}}{p_{2}} \Pi_{m, n+1}(x) \frac{f_{X_{n}}^{3}\left(X_{n+1}\right)}{f_{X_{n}}^{2}\left(X_{n+1}\right)} \mid\right. \\
&\left.X_{n-1}=t, X_{n}=u, \Pi_{n}^{1}(x)=\alpha, \Pi_{n}^{2}(x)=\beta, \Pi_{m n}(x)=\gamma\right) \\
&=\frac{q_{2}}{p_{2}} \gamma p_{2} E\left(\frac{f_{u}^{2}\left(X_{n+1}\right)}{H\left(u, X_{n+1}, \alpha, \beta\right)} \frac{f_{u}^{3}\left(X_{n+1}\right)}{f_{u}^{2}\left(X_{n+1}\right)} \mid \mathcal{F}_{n}\right) \\
& \stackrel{(10)}{=} q_{2} \gamma \int_{\mathbb{E}} \frac{f_{u}^{3}(s)}{H(u, s, \alpha, \beta)} H(u, s, \alpha, \beta) P_{u}(d s)=q_{2} \gamma
\end{aligned}
$$


and

$$
Q_{x} h(t, u, \alpha, \beta, \gamma)=\frac{q_{2}}{p_{2}} \gamma \max \left\{\frac{f_{t}^{3}(u)}{f_{t}^{2}(u)}, p_{2}\right\} .
$$

Define $r_{n}(t, u)$ as in the statement of the lemma. We show that

$$
Q_{x}^{l} h(t, u, \alpha, \beta, \gamma)=\frac{q_{2}}{p_{2}} \gamma r_{l}(t, u)
$$

for $l=1,2, \ldots$ By (15) we have $Q_{x} h=\left(q_{2} / p_{2}\right) \gamma r_{1}$. Assume (16) for $l \leq k$. By (10) we have

$$
\begin{gathered}
T_{x} Q_{x}^{k} h(t, u, \alpha, \beta, \gamma)=E_{x}\left(\frac{q_{2}}{p_{2}} \Pi_{m, k+1}(x) r_{k}\left(X_{n}, X_{n+1}\right) \mid\right. \\
\left.X_{n-1}=t, X_{n}=u, \Pi_{n}^{1}(x)=\alpha, \Pi_{n}^{2}(x)=\beta, \Pi_{m n}(x)=\gamma\right) \\
=\frac{q_{2}}{p_{2}} \gamma p_{2} \int_{\mathbb{E}} r_{k}(u, s) f_{u}^{2}(s) P_{u}(d s) .
\end{gathered}
$$

It is easy to show (see Shiryaev (1978)) that

$$
Q_{x}^{k+1} h=\max \left\{h, T_{x} Q_{x}^{k} h\right\} \quad \text { for } k=1,2, \ldots
$$

Hence we get $Q_{x}^{k+1} h=\left(q_{2} / p_{2}\right) \gamma r_{k+1}$ and (16) is proved for $l=1,2, \ldots$ This gives

and

$$
h^{*}(t, u, \alpha, \beta, \gamma)=\frac{q_{2}}{p_{2}} \gamma \lim _{k \rightarrow \infty} r_{k}(t, u)=\frac{q_{2}}{p_{2}} \gamma r^{*}(t, u)
$$

$$
\begin{aligned}
\eta_{m n} & =\operatorname{ess} \sup _{(\tau, \sigma) \in \mathcal{T}_{m n}} E_{x}\left(\xi_{\tau, \sigma} \mid \mathcal{F}_{m n}\right) \\
& =h^{*}\left(X_{n-1}, X_{n}, \Pi_{n}^{1}(x), \Pi_{n}^{2}(x), \Pi_{m n}(x)\right) .
\end{aligned}
$$

We have

$$
T_{x} h^{*}(t, u, \alpha, \beta, \gamma)=\frac{q_{2}}{p_{2}} \gamma p_{2} \int_{\mathbb{E}} r^{*}(u, s) f_{u}^{2}(s) P_{u}(d s)=\frac{q_{2}}{p_{2}} \gamma R^{*}(u)
$$

and $\sigma_{m}^{*}$ has the form (11). By (5) and (10) we obtain

$$
\begin{aligned}
\eta_{m}(x) & =f\left(X_{n-1}, X_{n}, \Pi_{n}^{1}(x), \Pi_{n}^{2}(x)\right)=E\left(\eta_{m, m+1} \mid \mathcal{F}_{m}\right) \\
& =E\left(\frac{q_{2}}{p_{2}} \Pi_{m, m+1} r^{*}\left(X_{m}, X_{m+1}\right) \mid \mathcal{F}_{m}\right) \\
& =\frac{q_{2}}{p_{2}} \Pi_{m m} \int_{\mathbb{E}} r^{*}\left(X_{m}, s\right) f_{X_{m}}^{2}(s) P_{X_{m}}(d s) .
\end{aligned}
$$

By Lemmas 4 and 3 the optimal stopping problem (6) has been transformed to the optimal stopping problem for the homogeneous Markov process

$$
W=\left\{\left(X_{m-1}, X_{m}, \Pi_{m}^{1}(x), \Pi_{m}^{2}(x)\right), m \in \mathbb{N}, x \in \mathbb{E}\right\}
$$


with the reward function

$$
f(t, u, \alpha, \beta)=\frac{q_{1} q_{2}}{p_{1} p_{2}} \cdot \frac{f_{t}^{2}(u)}{f_{t}^{1}(u)} \alpha R^{*}(u) .
$$

Lemma 5. The solution of the optimal stopping problem (6) for $n=$ $1,2, \ldots$ has the form

$$
\tau_{n}^{*}=\inf \left\{k \geq n:\left(X_{k-1}, X_{k}, \Pi_{k}^{1}(x), \Pi_{k}^{2}(x)\right) \in B^{*}\right\}
$$

where $B^{*}=\left\{(t, u, \alpha, \beta): f_{t}^{2}(u) / f_{t}^{1}(u) \geq p_{1} \int_{\mathbb{E}} v^{*}(u, s) P_{u}(d s)\right\}$. Here $v^{*}(t, u)$ $=\lim _{n \rightarrow \infty} v_{n}(t, u)$, where $v_{0}(t, u)=R^{*}(u)$ and

$$
v_{n+1}(t, u)=\max \left\{\frac{f_{t}^{2}(u)}{f_{t}^{1}(u)}, p_{1} \int_{\mathbb{E}} v_{n}(u, s) f_{u}^{1}(s) P_{u}(d s)\right\} .
$$

The function $v^{*}(t, u)$ satisfies the equation

$$
v^{*}(t, u)=\max \left\{\frac{f_{t}^{2}(u)}{f_{t}^{1}(u)}, p_{1} \int_{\mathbb{E}} v^{*}(u, s) f_{u}^{1}(s) P_{u}(d s)\right\} .
$$

The value of the problem is $V_{n}=v^{*}\left(X_{n-1}, X_{n}\right)$.

Proof. For any Borel function $u: \mathbb{E} \times \mathbb{E} \times[0,1]^{2} \rightarrow[0,1]$ define two operators

$$
\begin{aligned}
& T_{x} u(t, s, \alpha, \beta)=E_{x}\left(u\left(X_{n}, X_{n+1}, \Pi_{n+1}^{1}(x), \Pi_{n+1}^{2}(x)\right) \mid\right. \\
& \left.X_{n-1}=t, X_{n}=s, \Pi_{n}^{1}(x)=\alpha, \Pi_{n}^{2}(x)=\beta\right)
\end{aligned}
$$

and

$$
Q_{x} u(t, s, \alpha, \beta)=\max \left\{u(t, s, \alpha, \beta), T_{x} u(t, s, \alpha, \beta)\right\} .
$$

As in the proof of Lemma 4 we conclude that the solution of (6) is the Markov time

$$
\begin{aligned}
\tau_{m}^{*}=\inf \left\{n>m: f\left(X_{n-1}, X_{n}, \Pi_{n}^{1}(x), \Pi_{n}^{2}(x)\right)\right. \\
\left.=f^{*}\left(X_{n-1}, X_{n}, \Pi_{n}^{1}(x), \Pi_{n}^{2}(x)\right)\right\},
\end{aligned}
$$

where $f^{*}=\lim _{k \rightarrow \infty} Q_{x}^{k} f(t, u, \alpha, \beta)$. We have

$$
\begin{aligned}
& T_{x} h(t, u, \alpha, \beta)= E_{x}\left(\frac{q_{1} q_{2}}{p_{1} p_{2}} \Pi_{n+1}^{1}(x) \frac{f_{X_{n}}^{2}\left(X_{n+1}\right)}{f_{X_{n}}^{1}\left(X_{n+1}\right)} R^{*}\left(X_{n+1}\right) \mid\right. \\
&\left.X_{n-1}=t, X_{n}=u, \Pi_{n}^{1}(x)=\alpha, \Pi_{n}^{2}(x)=\beta\right) \\
&=\frac{q_{1} q_{2}}{p_{1} p_{2}} \gamma p_{1} E\left(\frac{f_{u}^{1}\left(X_{n+1}\right)}{H\left(u, X_{n+1}, \alpha, \beta\right)} \cdot \frac{f_{u}^{2}\left(X_{n+1}\right)}{f_{u}^{1}\left(X_{n+1}\right)} R^{*}\left(X_{n+1}\right) \mid \mathcal{F}_{n}\right)
\end{aligned}
$$




$$
\begin{aligned}
& \stackrel{(10)}{=} \frac{q_{1} q_{2}}{p_{1} p_{2}} \alpha p_{1} \int_{\mathbb{E}} \frac{f_{u}^{2}(s)}{H(u, s, \alpha, \beta)} H(u, s, \alpha, \beta) R^{*}(s) P_{u}(d s) \\
& =\frac{q_{1} q_{2}}{p_{1} p_{2}} \alpha p_{1} \int_{\mathbb{E}} R^{*}(s) f_{X_{n}}^{2}(s) P_{X_{n}}(d s)
\end{aligned}
$$

and

$$
\begin{aligned}
Q_{x} f(t, u, \alpha, \beta) & =\frac{q_{1} q_{2}}{p_{1} p_{2}} \alpha \max \left\{\frac{f_{t}^{2}(u)}{f_{t}^{1}(u)}, p_{1} \int_{\mathbb{E}} R^{*}(s) f_{u}^{2} P_{u}(d s)\right\} \\
& =\frac{q_{1} q_{2}}{p_{1} p_{2}} \alpha v_{1}(t, u) .
\end{aligned}
$$

We show that

$$
Q_{x}^{l} f(t, u, \alpha, \beta)=\frac{q_{1} q_{2}}{p_{1} p_{2}} \alpha v_{l}(t, u)
$$

for $l=1,2, \ldots$, where $v_{l}(t, u)$ is defined as in the statement of the lemma. By (23) we have $Q_{x} f=\frac{q_{1} q_{2}}{p_{1} p_{2}} \alpha v_{1}$. Assume (24) for $l \leq k$. By (10) we have

$$
\begin{gathered}
T_{x} Q_{x}^{k} f(t, u, \alpha, \beta)=E_{x}\left(\frac{q_{1} q_{2}}{p_{1} p_{2}} \Pi_{k+1}^{1}(x) v_{k}\left(X_{n}, X_{n+1}\right) \mid\right. \\
\left.X_{n-1}=t, X_{n}=u, \Pi_{n}^{1}(x)=\alpha, \Pi_{n}^{2}(x)=\beta\right) \\
=\frac{q_{1} q_{2}}{p_{1} p_{2}} \alpha p_{1} \int_{\mathbb{E}} v_{k}(u, s) f_{u}^{1}(s) P_{u}(d s) .
\end{gathered}
$$

Hence $Q_{x}^{k+1} f=\frac{q_{1} q_{2}}{p_{1} p_{2}} \alpha v_{k+1}$ and (24) is proved for $l=1,2, \ldots$ This gives

$$
f^{*}(t, u, \alpha, \beta)=\frac{q_{1} q_{2}}{p_{1} p_{2}} \alpha \lim _{k \rightarrow \infty} v_{k}(t, u)=\frac{q_{1} q_{2}}{p_{1} p_{2}} \alpha v^{*}(t, u)
$$

and

$$
V_{m}=\frac{q_{1} q_{2}}{p_{1} p_{2}} \Pi_{m}^{1} v^{*}\left(X_{m-1}, X_{m}\right)
$$

We have

$$
T_{x} f^{*}(t, u, \alpha, \beta)=\frac{q_{1} q_{2}}{p_{1} p_{2}} \alpha p_{1} \int_{\mathbb{E}} v^{*}(u, s) f_{u}^{1}(s) P_{u}(d s) .
$$

It follows that $\tau_{n}^{*}$ has the form (20). The value of the problem (6) and (3) is

$$
\begin{aligned}
v_{0}(x) & =E_{x}\left(V_{1} \mid \mathcal{F}_{0}\right)=\frac{q_{1} q_{2}}{p_{1} p_{2}} E_{x} \Pi_{1}^{1}(x) v^{*}\left(x, X_{1}\right) \\
& =\frac{q_{1} q_{2}}{p_{2}} \int_{\mathbb{E}} v^{*}(x, s) f_{x}^{1}(s) P_{x}(d s) .
\end{aligned}
$$

By Lemmas 4 and 5 the solution of the problem $D_{00}$ can be formulated as follows. 
THEOREM 4.1. The compound stopping time $\left(\tau^{*}, \sigma_{\tau^{*}}^{*}\right)$, where $\sigma_{m}^{*}$ is given by (11) and $\tau^{*}=\tau_{0}^{*}$ is given by (20) is a solution of the problem $D_{00}$. The value of the problem is

$$
P_{x}\left(\tau^{*}<\sigma^{*}<\infty, \theta_{1}=\tau^{*}, \theta_{2}=\sigma_{\tau^{*}}^{*}\right)=\frac{q_{1} q_{2}}{p_{2}} \int_{\mathbb{E}} v^{*}(x, s) f_{x}^{1}(s) P_{x}(d s) .
$$

Remark 1. The problem can be extended to optimal detection of more than two successive disorders. The distribution of $\theta_{1}, \theta_{2}$ may be more general. The general a priori distributions of disorder moments lead to more complicated formulae, since the corresponding Markov chains are not homogeneous.

\section{References}

T. Bojdecki (1979), Probability maximizing approach to optimal stopping and its application to a disorder problem, Stochastics 3, 61-71.

T. Bojdecki (1982), Probability maximizing method in problems of sequential analysis, Mat. Stos. 21, 5-37 (in Polish).

Y. Chow, H. Robbins and D. Siegmund (1971), Great Expectations: The Theory of Optimal Stopping, Houghton Mifflin, Boston.

G. Haggstrom (1967), Optimal sequential procedures when more than one stop is required, Ann. Math. Statist. 38, 1618-1626.

M. Nikolaev (1979), Generalized sequential procedures, Lit. Mat. Sb. 19, 35-44 (in Russian).

M. Nikolaev (1981), On an optimality criterion for a generalized sequential procedure, ibid. 21, 75-82 (in Russian).

A. Shiryaev (1978), Optimal Stopping Rules, Springer, New York.

K. Szajowski (1992), Optimal on-line detection of outside observations, J. Statist. Plann. Inference 30, 413-422.

M. Yoshida (1983), Probability maximizing approach for a quickest detection problem with complicated Markov chain, J. Inform. Optim. Sci. 4, 127-145.

Krzysztof Szajowski

Institute of Mathematics

Technical University of Wrocław

Wybrzeże Wyspiańskiego 27

50-370 Wrocław, Poland

E-mail: szajow@im.pwr.wroc.pl 